\title{
A New Distribution System Performance Approach to the Switch Allocation Problem Under Smart Grid Framework
}

\author{
Hatem Zaki ${ }^{1}$, R.A. Swief ${ }^{2}$, T.S. Abdel-Salam², M.A.M. Mostafa ${ }^{2}$ \\ ${ }^{1}$ Distribution System Planning, BC Hydro, Vancouver BC, Canada \\ ${ }^{2}$ Dept. of Electrical Power \& Machines, Ain Shams University, Cairo, Egypt
}

\begin{abstract}
This paper proposes a new vision of the Distribution System Switch Allocation problem considering new system performance measures. The mathematical model has been rebuilt with a new aggregated multi-objective formula, minimizing a newly developed performance index while achieving minimum annual energy lost. A new practical weighted combined system performance index, consisting of Reliability, Resiliency and Vulnerability, is applied and tested to be used by utilities replacing the common simple reliability index combination. The new model uses mixed integer design variables to determine the number, location and status of switches. A set of eight logical and technical constraints was applied to provide the best description of the real existing system constraints. A new algorithm of checking the system radial topology is also applied to the problem. The problem was solved using the Genetic Algorithm and was tested on a 54-bus real distribution test system, deemed more complicated than the test systems found in literature, to demonstrate its validity and effectiveness in real life systems.
\end{abstract}

\section{Introduction}

In today's decision making, under Smart Grid (SG) approaches, system performance is a vital characteristic of the Distribution System (DS) Planning problem and must be combined as part of the objectives when making such significant decision [1].

Switch Allocation (SA) is a crucial and attractive topic to power DS planners and operators. As presented by past researches, presence of switches in DS is nowadays essential, for the following three main goals:

(1) To sectionalize radial distribution feeders, for the purpose of limiting outage durations by limiting the number of customers out of service during faults [2],

(2) To tie more than one feeder for the purpose of creating open loop topologies to facilitate power restoration during contingencies [3], and

(3) To reconfigure the DS for minimization of active power (and energy) losses in the DS during normal operations [4].

While the first and the second goals play a role only in improving the System Performance (SP), the third goal will impact the System Operational Flexibility (SOF) in addition to overall system financial performance.

Strategically selecting the location of switches in the DS and, perhaps automating some or all of them, plays a role in maximizing the value of certain objectives [5]. Commonly used objectives, in past research, often included maximization of reliability [6], minimization of switch capital and running costs [7], minimization of cost of outages [8], minimization of total Expected Energy Not Served (EENS) and minimization of system losses
[4]. Combinations of these objectives have been used in the past as the main interest of researchers around the subject of SA in DSs. Even though these past researches used EENS as an objective to express the SP, it seems that utilities interest is more focused towards common performance indices. These common indices include System Average Interruption Frequency Index (SAIFI), System Average Interruption Duration Index (SAIDI), percentage of Customers Experiencing Multiple Interruptions of 4 or more (CEMI-4), and Customers Experiencing Lengthy Interruption Durations of 6 or more hours (CELID-6) [9].

In this paper, a new formulation of the DS model for the purpose of SA including a new combinatorial performance index is proposed and demonstrated using test cases. The proposed index addresses utility concerns and is proven to be a good indicator of the system performance. The paper neither discusses, nor proposes the automation of switches in the DS but rather 1ocuses on presenting the proposed model with its application and results.

Previous research on finding the optimal placement and the number of switches in DS involved several solution methodologies. These methodologies included analytical hierarchical process decision making algorithm [10], heuristics [11], immune [12], reactive tabu search [6] or decomposition approach [13]. Even though good results have been obtained from these methodologies, they only applied a smaller number of constraints. These constraints are necessary to make the system simulate the real-world applications. 
The solution methodology used in this paper is the Genetic Algorithm (GA) with modifications necessary to obtain the best solution of the SA problem. A list of eight constraints has been applied and divided into two main groups to better simulate the real-world systems and make the proposed methodology useful for planners and operators. A new approach to one of the constraints has also been applied to ensure the usefulness of the obtained results.

The paper is divided into six sections. In addition to this introduction, the new model description is presented in section II. The mathematical formulation representing the proposed model is discussed in section III and the solution algorithm is described in section IV. Test cases and conclusion are presented in sections $\mathrm{V}$ and $\mathrm{VI}$ respectively.

\section{Problem description}

On the roadway of using multiple objectives as targets for locating switches, this paper proposes the use of an aggregated sum of two components: (A) a new combinatorial performance index formed of three common performance indices, and $(B)$ the system annual energy losses. The goal of the problem is to minimize the aggregated sum of both objectives.

\subsection{The new combinatorial performance index}

Even though there is a clear need for an expressive system performance index under the smart Grid framework, neither regulators nor utilities were able to agree on a complete standard understanding of how to represent system performance. Standard reliability indices have been used and proposed by IEEE with a later improvement to include resiliency to cover expected large environmental impacts of systems [9]. Most utilities have combined these indices in one formula with weighted sums tailored to their needs [14].

Following this trend, the proposed Contingency Index (CONDEX), in this paper, is formed of three components:

i. The Unified Reliability Index (URI) - This index has been often used to express reliability of a given DS with historical outages. URI can be formed of the weighted sum of multiple known reliability indices [14]. In this paper, and for practical reasons, only four indices were used, which are SAIFI, SAIDI, CEMI-4 and CELID-6. Other indices require special equipment to obtain data which is usually not available for most utilities due to their significant costs.

ii. The System Resiliency Index (SRI) - As proposed by the IEEE guideline [9], this index is CELID-12, which reflects the percentage of customers experiencing 12 or more hours of outages. Many utilities have modified this index to include the number of years, where CELID-12 has happened, over the study period. In this paper, the new modified SRI, including the number of years, is used.

iii. System Vulnerability Index (SVI) - This is the new index presented in this work. SVI represents the ability of the DS to stay in-service during and after a massive disaster such as a massive earthquake, a one of a kind storms with destructive wind speed (not annual storms), large permanent floods, etc. To use a predictive Vulnerability index three weighted arguments are created and selected to form the SVI. This performance index is a function of the following arguments:

- Node Distance Index (NDI) which presents the distance between each node and its source (substation in most cases).

- Node Failure Rate Index (NFRI) which presents the failure rate of each node route as linked to its source.

- Node Failure Duration Index (NFDI) which presents the failure duration of each node route as linked to its source.

SVI is then formed of the sum of the weighted values of NDI, NFRI, and NFDI.

CONDEX is hence formed of the weighted sum of URI, SRI and SVI. The presence of these weights provides enough flexibility to adjust the system configuration according to the highest priority index according to the strategy of the utility.

\subsection{The system annual energy losses}

In determining energy losses many researches have approximated the daily load curve to off-peak and peak averages and have taken seasonal annual averages to represent annual loads [15]. In modern systems, if digital monitoring devices are used, obtaining hourly annual loads, and hence a load factor $(L F)$ is possible.

The Loss factor can then be determined using the common approximate formula created by Buller and Woodrow [16].

Where,

$$
F L S=(1-k) * L F+k * L F^{2}
$$

FLS is the Loss Factor to be determined, $k$ is an imperical constant

$k$ can be determined using examination of circuits of a certain utility and if not known it can be assumed 0.85 [17]. The total Annual Energy Losses (AEL) can then be determined using the following formula:

$$
A E L=\text { Peak Power Loss } * 8760 * \text { FLS }
$$

The Peak Power Loss can be found from the load flow of each line section. The 8760 is the total number of service hours per year. Each load can be represented by its own annual load shape as was done in the EPRI Green Circuits project.

In this paper, annual hourly loads were used for the study systems to determine annual energy delivered. It could be safely assumed that this annual load curve is standard and that the system will follow this annual load curve every year.

AEL of a DS is a function of loads and can be calculated using load flow for each line section. FLS can be calculated using equation (1). Then AEL can be determined for each line section using equation (2). Based on this formula, the system will have a different AEL for each configuration depending on line section 
status. AEL can also be expanded to reflect growth in energy losses every year for all years of the study period.

The above-mentioned Objectives are subjected to a number of constraints. These constraints limit the optimum solution to a practical implementable solution, making the model as close as possible to the systems implemented in real life. These constraints are explained further in the Mathematical Formulation section.

\section{Mathematical formulation}

As mentioned in the Problem Description section, the objective function is formed of two parts aggregated and minimized under one representation. To avoid unit and number compromise in the objective function, the two parts of the objective function have been normalized by referring them to the maximum values in the system. While CONDEX is referred to the maximum value, $A E L$ is referred to the total energy required by the system. This way AEL becomes comparable to CONDEX as it will always be less than 1 .

As such the mathematical minimization problem can simply be as follows:

$$
\text { Minimize } F=\text { CONDEX }+A E L
$$

Equation (3) describes the overview of the objective function. The components of the Objective function consist of two parts, in addition to the constraints. The details are described in the following subsections.

\subsection{Formulation of contingency index (CONDEX)}

CONDEX is the first objective and its formula is as follows:

Where, CONDEX $=A * U R I+B * S R I+C * S V I$

$A$ is the weighting factor of the Unified Reliability Index (URI) $B$ is the weighting factor of the System Resiliency Index (SRI) $C$ is the weighting factor of the System Vulnerability Index (SVI) $i$ - URI is mathematically defined as:

$$
\begin{gathered}
U R I=a 1 * S A I F I+a 2 * S A I D I+a 3 * C E M I-4 \\
+a 4 * C E L I D-6
\end{gathered}
$$

Where,

$a 1, a 2, a 3, \& a 4$ are the weighting factors of each reliability index

SAIFI is the reliability Index known as System Average Inerruption Frequency Index

SAIDI is the reliability Index known as System Average Interruption Duration Index

CEMI - 4 is Customers Experiencing Multiple Interruptions

CELID of 4 or more

-6 is percentage of number of Customers Experiencing Lengthy Interruption Durations of 6 hours or more ii- $S R I$ is mathematically defined as:

Where,

$$
S R I=C E L I D-12+\text { NyrS }
$$

CELID - 12 is the percentage of Customers Experiencing Lengthy Interruption Durations of 12 hours or more for a given number of years

Nyrs is the ratio of number of years there has been 12 hours

or more outages during the span of given number of years CELID - 12 has been applied

It is common to use 5 years for most cases as the ultimate number of years for system resiliency measurement.

iii- SVI is mathematically defined as:
Where,

$c 1, c 2, c 3$ are the weighting factors of each vulnarability index

$N D I, N F R I$ and $N F D I$ are as previously defined in the Problem Description section.

\subsection{Formulation of the annual energy lost (AEL)}

As indicated in the Problem Description section AEL can be calculated for each individual line section. Therefore, the system AEL can be as follows:

Where,

$$
A E L=\sum_{t=1}^{T} \frac{1}{E_{s t}} \sum_{i=1}^{m} P_{L i}{ }^{(t)} * 8760 * F L S
$$

$m$ is the total number of line sections

$T$ is the total number of study period in years

$P_{L i}{ }^{(t)}$ is the Peak Power Lost of section $i$ in year $t$ $E_{s t}$ is the Energy required by the system in year $t$ $F L S$ is calculated from the formula shown in equation (1) as mentioned above.

\subsection{Problem constraints}

The above-mentioned objectives are subjected to a number of constraints. These constraints consist of two sets of constraints: (a) Logical and, (b) Technical as follows:

(a) Logical Constraints:

i. Radial Structure - After isolating all power sources except substations, from the system under study, this constraint algorithm then performs two checks on the given system: (1) Internal Loops and (2) connectivity to supply. Fig. 1 shows an overview of the proposed radial structure algorithm.

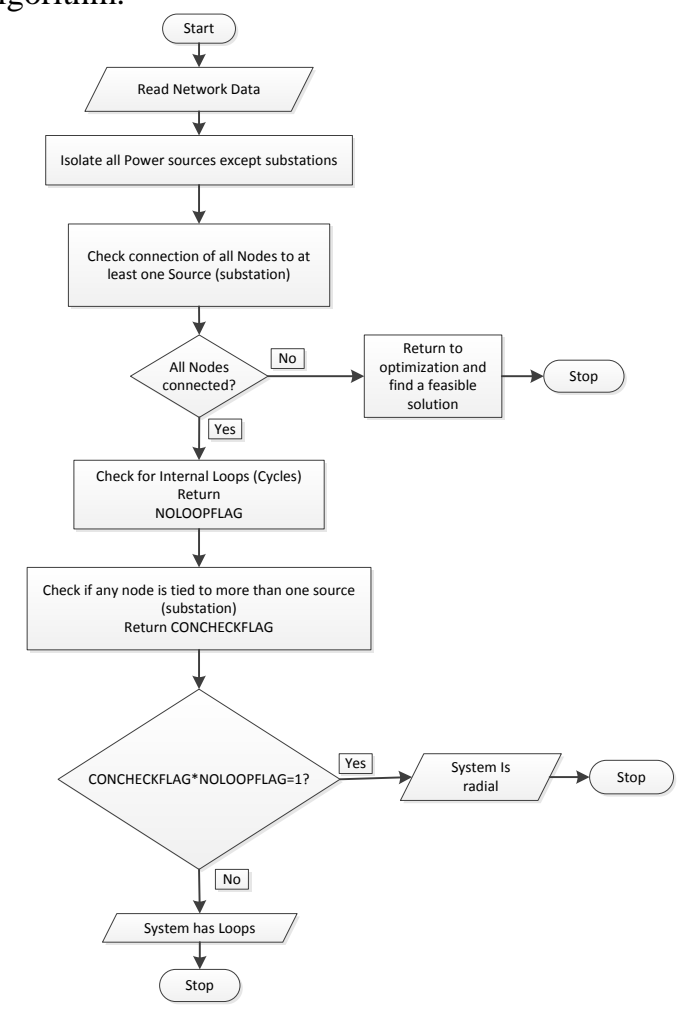


Fig. 1. Radial Structure Checking Algorithm overview.

The algorithm returns a flag (Radialflag), which returns 1 if the system is radial or 0 if the system contains loops or supplied by multiple substations.

ii. Node Connectivity - This is also implemented by an algorithm returning a flag called (concheck).

iii. Limit number of Normally Opened (NO) switches per feeder. This constraint can also be replaced with a cost limiting constraint to limit the total costs of switches being proposed in the system.

(b) Technical Constraints:

i. Voltage Limits Constraints - Voltages of all system nodes must be within standard ranges between a minimum value and maximum value as shown in inequality (9).

$$
V_{\text {min }} \leq V_{i} \leq V_{\text {max }}, i=1,2,3 \ldots, n
$$

Where,

$$
\begin{gathered}
n \text { is the total number of nodes not including } \\
\text { source nodes } \\
V_{\min } \text { and } V_{\text {max }} \text { are the standard allowable } \\
\text { voltage limits } \\
V_{i} \text { is the voltage of node } i
\end{gathered}
$$

iii. Substation Active Power Balance constraint assuming no reactive power limit, shown by equation (10)

$$
\sum_{i=1}^{n-s u b} P_{i} \leq P_{\max }^{(S)}
$$

Where,

$$
\begin{gathered}
n-s u b \text { is the total number of nodes connected } \\
\text { to a substation } S \\
P_{\text {max }}^{(S)} \text { is the maximum Active Power rating } \\
\text { of substation } S \\
P_{i} \text { is the active power of node } i \\
\text { connected to substation } S
\end{gathered}
$$

iv. Distributed Generation Power Balance constraint assuming no reactive power limit, shown by equation (11)

$$
\sum_{j=1}^{n g} P G_{j} \leq P G_{\max }^{(G)}
$$

Where,

$$
\begin{gathered}
n g \text { is the total number of Line sections } \\
\text { emerging from generator } G \\
P G_{\text {max }}^{(G)} \text { is the maximum Active Power rating } \\
\text { of generator } G \\
P G_{j} \text { is the active power of Line section } j \\
\text { emerging from generator } G
\end{gathered}
$$

v. Line section current thermal limits, shown in inequality (12)

$$
\begin{aligned}
& \text { Where, } \\
& \quad I_{i} \leq I_{\max } \\
& m \text { is the total number of line sections } \\
& I_{\text {max }} \text { is the line section conductor } \\
& \text { allowable current thermal limit } \\
& I_{i} \text { is the line section current flow }
\end{aligned}
$$

In past research, only a few of these constraints were applied to the SA problem. In this paper, all the abovementioned constraints were successfully applied producing good results.
The solution methodology of the SA problem, modeled in this paper is a robust approach to avoid long processing times, often found in past methodologies. The algorithm can be summarized in the following steps:

Step 1 - starts by determining if a line section is a lateral. If the line section is not a lateral it is a candidate for either a NO or a Normally Closed (NC) switch. In this step, laterals are also, reduced to their equivalent nodes. The system model is then rebuilt without laterals.

Step 2 - This step is similar to the reconfiguration problem solution. After determining candidates of system switches, the algorithm determines the best configuration of the DS using the identified two objectives mentioned in equations (4) and (8) including all logical and technical constraints. The system configuration, in this paper is determined using the GA.

Step 3 - the proposed number of switches and their locations then become the NO line sections found by the algorithm. These switches represent the optimum locations that will minimize the objectives for the given system, for the given study period.

Step 1 saves processing time by simplifying the system using elimination of laterals from the system to be studied. It prepares the problem for step 2 by rebuilding the model of the study system with switches only. Laterals are represented by their total load at the branching node. Step 2 then solves a simplified problem without laterals, rather than the traditional complex reconfiguration problem that include laterals in a separate constraint [18]. This system reduction results in reducing processing time.

Fig. 2 gives an overview of the solution algorithm.

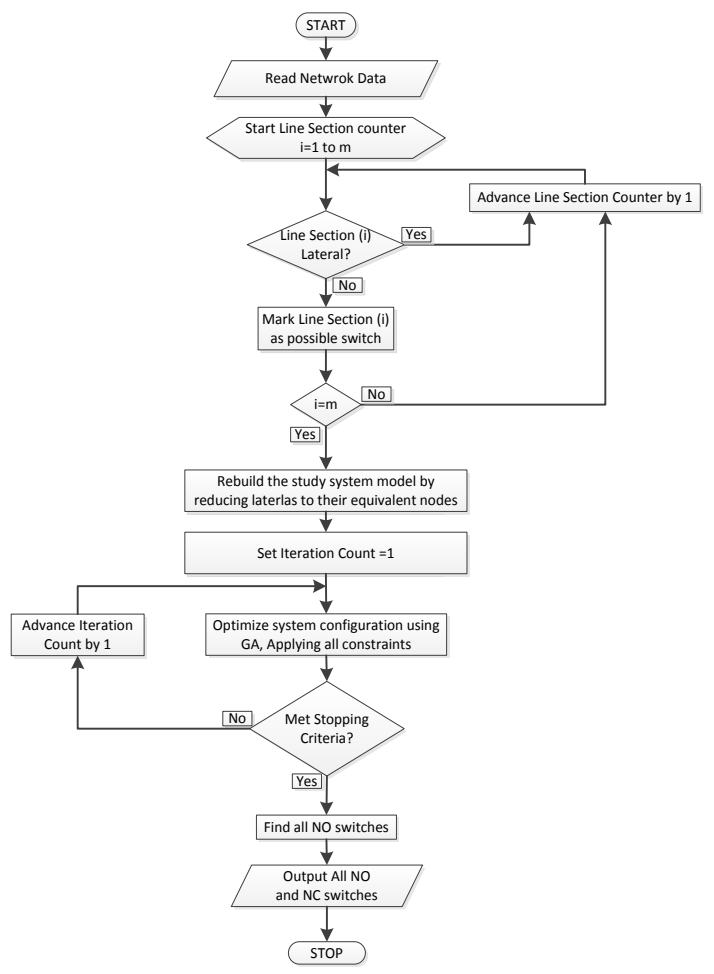

Fig.2. The SA solution Algorithm.

\section{Solution methodology}


The solution of the optimization problem was obtained using the GA, which is an older algorithm that appeared in the early 1990s [19]. GA (Goldberg 1989) is a search algorithm based on the principle of natural genetics and evolution.

The GA encompasses stopping criteria, which determines when to stop. This includes reaching maximum iterations, obtaining a solution that meets maximum tolerance in comparison to the previous solution, reaching maximum number of population generations, etc.

GA has proven to be a useful approach to address a wide a variety of optimization problems. Being a population-based approach, GA is well suited to solve the multi-objective optimization problems.

GA detailed explanation could be found in many references and therefore it will not be detailed further in this paper.

\section{Case study}

Several test cases were used to test the proposed model and solution algorithm. The 54-bus real system test case is presented here and discussed in this paper.

This test case consists of 54 buses, including 4 substations and 60 line-sections connecting these buses. The system also includes 2 DGs connected to two different buses and 7 laterals. These DGs are simulated using a P-V model with the assumption of infinite reactive power (VAR) abilities. Fig. 3 presents the test system with its original configuration.

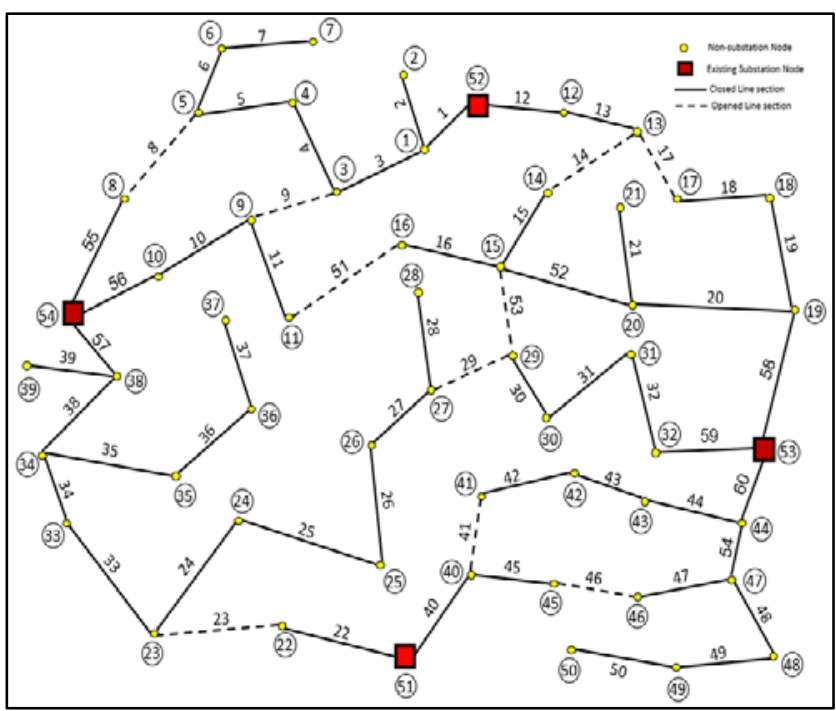

Fig. 3. 54-bus test system - original configuration.

DGs are tied to nodes 4 and 50 with installed capacities of 5 and $10 \mathrm{MW}$ respectively. The total load of the whole system is $4750 \mathrm{MW}$ with an average power factor of 0.95 .

Line-sections shown in dashed lines are existing NO sections. The test case result comparison is shown in Table 1 . In this test case, the $A, B$, and $C$ weighting factors stated in equation (4) are taken as unity.
Table 1. Test Case parameters before and after optimization.

\begin{tabular}{|l|c|c|}
\hline & $\begin{array}{c}\text { Before optimization } \\
\text { (original } \\
\text { configuration) }\end{array}$ & After optimization \\
\hline URI & 26.4748 & 8.836 \\
\hline \multicolumn{1}{|c|}{$S R I$} & 3.4433 & 3.1382 \\
\hline \multicolumn{1}{|c|}{ SVI } & 5.4 & 4.2336 \\
\hline$A E L(\mathrm{kWh})$ & $601,865.5$ & $436,746.88$ \\
\hline $\begin{array}{l}\text { Total } \\
\text { Objective } \\
\text { (F) }\end{array}$ & 162.0273 & 107.81 \\
\hline NO sections & $\begin{array}{c}8,9,14,17,23,29,41, \\
46,51,53\end{array}$ & $\begin{array}{c}3,5,14,17,27,33,42,47, \\
51,53\end{array}$ \\
\hline
\end{tabular}

Objective function parameters, such as URI, SRI and $S V I$, are not expected be individually reduced; rather the total objective function value will be reduced. In this example the proposed solution algorithm was also able to reduce each individual parameter. This is usually impacted by the weighting factors, mentioned in equations (4) through (7), given to these parameters. These weighting factors are determined according to the strategy of each utility to identify the priority parameters driving investments.

From Table 1 the solution proposes the use of a new set of NO switches. It is also clear that the solution algorithm has avoided placing switches on laterals such as line-sections 6 and 7, as they were eliminated during the solution application.

\section{Conclusion}

In this paper a new model for the SA problem was proposed. The new model included a combination of three performance indicators replacing the traditional EENS function and combined with the commonly used AEL function as a multi-objective function aggregated under one representation. Eight constraints were used in the solution for the first time. The new proposed model demonstrates its viability to arrive to an optimum solution considering the modern approaches of smart grids including performance. After testing the model using test systems with variable parameters it can be concluded that the model is a practical implementable model that proposes a solution suitable for finding a trade-off between performance and losses. The model can be easily applied in utilities and is recommended to be used by planners to help make the best investment decisions

\section{References}

1. M. Y. Wu, M. I. Ridzuan and S. Z. Djokic, "Smart grid functionalities for improving reliability of rural electricity networks," in Mediterranean Conference on Power Generation, Transmission, Distribution and Energy Conversion (MedPower), Belgrade, Serbia, (2017)

2. S. Ray, S. Bhattacharjee and A. Bhattacharya, "Optimal Allocation of Remote Control Switches in Radial distribution netwrok for Reliability 
Improvement," Ain Shams Engineering Journal Elsevier B.V., (2016)

3. H. Hashemi-Dezaki, H. Haeri-Khiavi and $H$. Askarian-Abyaneh, "Reliability optimization of Electrical Distribution Systems using Internal Loops to minimize Energy Not-Supplied (ENS)," Journal of Applied Reasearch and Technology, (2014)

4. J. C. Lopez, J. F. Franco and M. J. Rider, "Optimization-Based Switch Allocation to Improve Energy Losses and Service Restoration in Radial Electrical Distribution Systems," IET Generation, Transmission \& Distribution Journals, 10, 27922801 (2016)

5. I. Lim, T. S. Sidhu, M. S. Choi, S. J. Lee and B. N. Ha, "An Optimal Composition and Placement of Automatic Switches in DAS," IEEE Transactions on Power Delivery, 28, 1474-1482 (2013)

6. L. G. W. da Silva, R. A. F. Pereira and J. R. S. Mantovani , "Optimized Allocation of Sectionalizing Switches and Control and Protection Devices for Reliability Indices Improvement in Distribution Systems," in IEEE/PES Transmision and Distribution Conference and Exposition: Latin America (IEEE Cat. No. 04EX956), (2004)

7. L. Zemite, M. Gorobecs, A. Smats, A. Jasevics and A. Levchenkovs, "Genetic Algorithm for Optimization of Power Switch Allocation in Distribution Network," in IEEE International Conference on Environment, Electrical Engineering, Industrial and Commercial Power Systems, Europe (EEEIC / I\&CPS Europe) , (2017)

8. G. D. Ferreira, D. S. Gazzana, A. S. Bretas, A. L. Bettiol, A. Carniato, L. F. do N. Passos, R. Z. Homma and F. N. Molina, "Multiobjective MILP Model for Optimal Allocation of Automated Switching Devices in Electric Power Distribution Systems," in Poweer Tech, IEEE Eindhoven Conference, (2015)

9. "IEEE std. 1366-2012 IEEE Guide for Electric Power Distribution Reliability Indices," IEEE Power \& Energy Society, (2013)

10. D. P. Bernardon, M. Sperandio, V. J. Garcia, L. N. Canha, A. D. R. Abaide and E. F. B. DazaG, "AHP decision-making algorithm to allocate remotely controlled switches in distribution networks," IEEE transactions on Power Delivery, 26, 1884-1892 (2011)

11. A. Moradi and M. Fotuhi-Firuzabad, "Optimal switch placement in distribution systems using trinary particle swarmoptimization algorithm," IEEE Transactions on Power Delivery, 23, 271-279 (2008)

12. J. Chen, M. Peng, X. Gao and G. Li, "Multiobjective Distribution Network Planning Considering Invulnerability," in IEEE 2nd Information Technology, Networking, Electronic and Automation Control Conference (ITNEC), Chengdu, China, (2017)

13. P. M. S. Carvalho, L. A. F. M. Ferreira and A. J. C. da Silva, "A decomposition approach to optimal remote controlled switch allocation in distribution systems," Transactions on Power Delivery, 20, 1031-1036 (2005)

14. H. Sindi and E. El-Saadany, "Unified Reliability Index Development for Utility Performance Assessment," Intelligent Industrial Systems, 2, 149161 (2016)

15. Z. Ghofrani-Jahromi, M. Kazemi and M. Ehsan, "Distribution Switches Upgrade for Loss Reduction and Reliability Improvement," IEEE Transactions on Power Delivery, 30, 684-692 (2015)

16. F. H. Buller and C. A. Woodrow, "Load factorEquivalent hour values compared," Electricity World 92, 59-60 (1928)

17. R. F. Arritt, R. C. Dugan and T. A. Short, "Determining Loss Factor With the Use of Sequential-Time Simulations," IEEE Transactions on Industry Applications, 51, 1933-1937 (2015)

18. A. Mazza, G. Chicco, A. Russo and E. O. Virjoghe, "Multi-Objective Distribution Network Reconfiguration Based on Pareto Front Ranking," Springer Science \& Business Media, Intelligent Industrial Systems, Vols. DOI 10.1007/s40903-0160065-6, (2016)

19. V. Miranda, J. Ranito and L. Proenca, "Genetic algorithms in optimal multistage distribution network planning," IEEE Transactions on Power Systems, 9, 1927-1933 (1994) 